\title{
Is there a relationship between venous insufficiency and knee osteoarthritis?
}

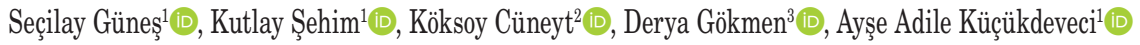 \\ ${ }^{1}$ Department of Physical Medicine and Rehabilitation, Ankara University School of Medicine, Ankara, Turkey \\ ${ }^{2}$ Department of Surgery, Division of Vascular Surgery, Ankara University School of Medicine, Ankara, Turkey \\ 3Department of Biostatistics, Ankara University School of Medicine, Ankara, Turkey
}

Received: July 30, 2019 Accepted: November 17, 2019 Published online: March 03, 2020

\begin{abstract}
Objectives: This study aims to evaluate whether there is a relationship between venous insufficiency (VI) and knee osteoarthritis (OA).

Patients and methods: Between February 2012 and May 2013, a total of 206 knees of 103 participants (14 males, 89 females; mean age $48.6 \pm 8.6$ years; range, 30 to 69 years) were enrolled. The study group included 59 patients who were diagnosed with knee OA and the control group included 44 healthy volunteers without any complaint in their knee joints. Demographic and clinical characteristics of all participants were recorded. Venous system of the lower extremities was evaluated by Doppler ultrasonography. All knees were evaluated using conventional radiography based on the Kellgren-Lawrence (K\&L) grading system and ultrasonography. Pain severity was evaluated using Likert pain scale and functioning was evaluated using Western Ontorio and McMaster Universities Osteoarthritis Index (WOMAC).

Results: Venous insufficiency was detected in $40.6 \%$ of the OA group and $15.9 \%$ of the control group (p=0.007). There was no statistically significant difference in the cartilage thicknesses and K\&L grading regarding the presence of VI ( $>0.05$ ); however, the percentage of the radiographic medial tibial sclerosis was higher in patients with VI (60\%) in the OA group (p>0.05). The WOMAC total scores were similar in both groups, while the WOMAC pain scores were higher in the patients with deep VI ( $>0.05)$.

Conclusion: Increased radiographic medial tibial sclerosis and higher WOMAC pain scores in patients with venous involvement in OA may lead to the hypothesis that venous system pathologies can affect the intraosseous microenvironment of the bone, resulting in pain and early subchondral bone involvement, consequently presenting as subchondral sclerosis.
\end{abstract}

Keywords: Cartilage, knee osteoarthritis, subchondral bone, venous insufficiency.

Osteoarthritis (OA) is defined as a disease primarily characterized by cartilage degeneration; however, in recent years, it is considered a joint failure involving the entire joint such as cartilage, synovium, subchondral bone, and ligamentous tissue..$^{[1,2]}$ The pathophysiology of the OA has not been clearly understood yet and the knowledge of vascular circulatory disturbances and their consequences to subchondral bone microenvironment is limited. It has been shown that blood vessels are located more particularly at the bone resorption sites ${ }^{[3]}$ and any alterations in bone perfusion result in hypoxia in the subchondral bone and also cause intraosseous hypertension and increased bone resorption. ${ }^{[4]}$ Increased subchondral bone resorption is associated with subchondral bone sclerosis and cartilage thinning. ${ }^{[5]}$

Osteoarthritis and chronic venous disease share the common risk factors such as obesity and prolonged standing. Chronic venous disease, particularly venous insufficiency (VI), is accompanied by venous hypertension. There are some studies assessing the contribution of circulatory disturbances in the pathophysiology of OA; however, the number of studies investigating the relationship between peripheral VI and $\mathrm{OA}$ is limited. ${ }^{[6]}$

Corresponding author: Seçilay Güneş, MD. Ankara Üniversitesi Tıp Fakültesi Fiziksel Tıp ve Rehabilitasyon Anabilim Dalı, 06230 Sıhhiye, Ankara, Türkiye.

e-mail: secilaygunes@ankara.edu.tr 
In the present study, we aimed to investigate whether there is a relationship between knee OA and VI.

\section{PATIENTS AND METHODS}

In this case-control study, all participants were selected among the patients who were admitted to the Outpatient Clinic of Physical Medicine and Rehabilitation, Ankara University School of Medicine between February 2012 and May 2013. A total of 206 knees of 103 participants (14 males, 89 females; mean age $48.6 \pm 8.6$ years; range, 30 to 69 years) were evaluated for the study. The study population included 59 patients who were diagnosed with knee OA according to the clinical and radiological American College of Rheumatology (ACR) knee OA criteria. ${ }^{[7]}$ Forty-four healthy volunteers without any complaint in their knee joints were selected as the control group. Individuals with intraarticular injection within the previous six months, previous knee surgery, previous lower extremity vascular surgery, cardiorespiratory disease, acute arterial or venous thrombosis, inflammatory arthritis, intraarticular neoplasms, and trauma were excluded from the study. A written informed consent was obtained from each participant. The study protocol was approved by the Ankara University School of Medicine Ethics Committee (No. 01-48-12). The study was conducted in accordance with the principles of the Declaration of Helsinki.

Data including demographic and clinical characteristics of all participants such as age, gender, marital status, body mass index, and history of deep venous thrombosis were recorded.

\section{Venous insufficiency evaluation}

Venous Doppler ultrasonography was performed in all lower extremity peripheral veins of the participants by an experienced and blinded vascular surgeon (CK) using a 7 to $12 \mathrm{MHz}$ linear probe (HDI 5000, Philips Medical Systems, Bothell, WA, USA). Lower extremities were examined in the standing position on the examination table. Valsalva maneuver was performed after the normal evaluation, and VI was recorded if there was an abnormal retrograde outflow during Valsalva maneuver. A reflux time of $>0.5 \mathrm{sec}$ for superficial veins and $1.0 \mathrm{sec}$ for deep veins is typically used to diagnose the presence of reflux. ${ }^{[8]}$

Bilateral common femoral, femoral, deep femoral, popliteal, anterior tibial, posterior tibial, long saphenous, short saphenous, and perforating veins were examined. The clinical status of venous disease was evaluated using clinical, etiological, anatomical and pathological classification (CEAP) system which is based on clinical manifestations (C), etiological factors (E), anatomic distribution of disease (A), and underlying pathophysiological findings (P). ${ }^{[9]}$ The clinical classification has seven categories (0-6) and is further categorized by the presence or absence of symptoms. The etiological classification is the basis on congenital, primary, and secondary causes of venous dysfunction. The anatomic classification describes the superficial, deep, and perforating venous systems, with multiple venous segments that may be involved. The pathophysiological classification describes the underlying mechanism resulting in chronic VI, including reflux, venous obstruction, or both (Figure 1).

Common femoral, femoral, long saphenous, short saphenous vein involvement was considered superficial VI, while the involvement of deep femoral, popliteal, anterior tibial, posterior tibial and perforating veins was considered deep VI.

\section{Radiographic evaluation of the knees}

At baseline, all participants underwent bilateral weight-bearing anteroposterior knee radiographs. Radiographs of the knees were evaluated according to the Kellgren-Lawrence $(\mathrm{K} \& \mathrm{~L})$ grading scale ${ }^{[10]}$ by an experienced physician. This scale evaluates knees according to joint space narrowing (JSN) and presence of osteophyte: Grade 0 no radiographic features of OA are present; Grade 1 there is doubtful JSN and possible osteophytic lipping; Grade 2 definite osteophytes and possible JSN is present; Grade 3 multiple osteophytes, definite JSN, sclerosis, possible bony deformity is seen; Grade 4 large osteophytes, marked JSN, severe sclerosis and definitive bony deformity is present. The presence of medial tibial subchondral sclerosis on the anteroposterior knee radiography was evaluated separately in all individuals, since venous system involvement may be an indicator of subchondral bone involvement.

\section{Ultrasonographic evaluation of the knees}

Cartilage thickness measurements were made with a linear probe (7-12 MHz Logiq P5, GE Medical Systems, CA, USA). The patients were examined in the supine position on the examination table with their knees in maximum flexion and the probe was placed in an axial position on the suprapatellar area where the superior origin of patella finished. ${ }^{[1]]}$ The distal femoral cartilage was visualized as a strongly 


\begin{tabular}{|c|c|}
\hline Clinical manifestations \\
\hline C0: & No visible or palpable signs of venous disease. \\
C1: & Telangiectasis or reticular veins. \\
C2: $\quad$ Varicose veins; distinguished from reticular veins by a diameter of 3 mm or more. \\
C3: $\quad$ Edema. \\
C4: $\quad$ Changes in skin and subcutaneous tissue secondary to CVD, now divided into \\
C4a: $\quad$ Pigmentation or eczema. \\
C4b: Lipodermatosclerosis or atrophie blanche. \\
C5: $\quad$ Healed venous ulcer. \\
C6: & Active venous ulcer. \\
\hline Etiologic classification \\
\hline Ec: $\quad$ Congenital \\
Ep: $\quad$ Primary \\
Es: $\quad$ Secondary (post thrombotic) \\
En: $\quad$ No venous cause identified \\
\hline Anatomic classification \\
\hline As: $\quad$ Superficial veins \\
Ap: $\quad$ Perforator veins \\
Ad: $\quad$ Deep veins \\
An: $\quad$ No venous location identified \\
\hline Pathophysiologic classification \\
\hline Pr: & Reflux \\
Po: & Obstruction \\
Pr,o: & Reflux and obstruction \\
\hline Figure & No venous pathophysiology identifiable \\
\hline
\end{tabular}

anechoic structure between the sharp bony cortex and the suprapatellar fat. Three (mid-point) measurements were taken from each knee, at the lateral femoral condyle (LFC), intercondylar area (ICA), medial femoral condyle (MFC) (Figure 2). Each distance was measured three times in total and the average measurement was calculated.

\section{Evaluation of pain and functioning}

Functioning was evaluated using the Turkish version of Western Ontario and McMaster
Universities Osteoarthritis Index (WOMAC 3.0). ${ }^{[12]}$ The WOMAC assesses functioning in three subscales: pain (5 items), joint stiffness (2 items), and physical function (17 items) through 24 questions (0 to 100 visual analog scale). Each item is scored as 0-4: none (0), mild (1), moderate (2), severe (3), and extreme (4). The scores for each subscale are summed up, with a possible score range of $0-20$ for pain, $0-8$ for stiffness, and 0-68 for physical function, and total score changes $0-100$. High scores indicate increased pain and stiffness and deterioration in physical

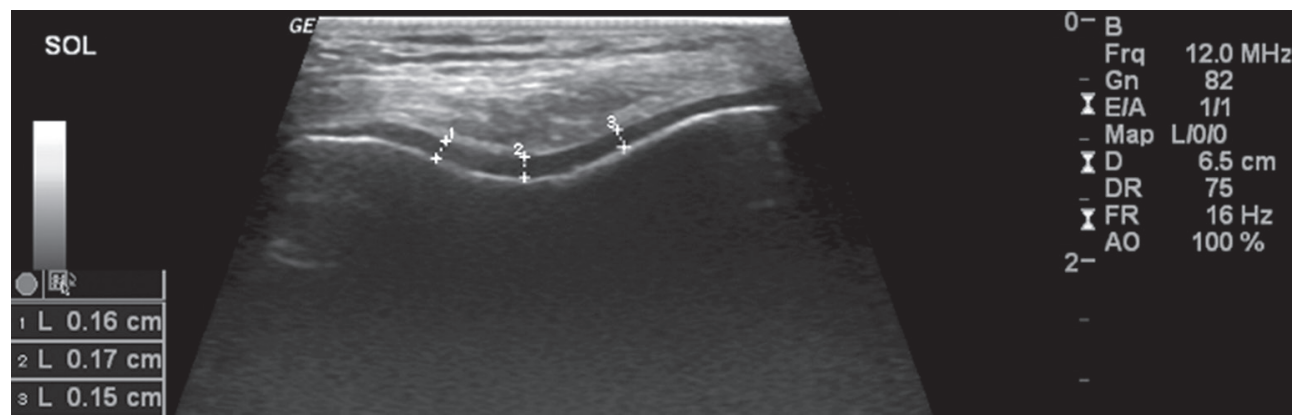

Figure 2. An ultrasonographic view of cartilage thickness measurement. 
function. The Likert pain scale was used for rating pain severity, and higher values indicates increased pain severity. ${ }^{[1-5]}$

\section{Statistical analysis}

All statistical analyses were performed using the RStudio Version 0.96.122 (RStudio: Integrated Developement for R. RStudio, Inc., Boston, MA, USA). Descriptive data were expressed in mean \pm standard deviation (SD), median (min-max), and number and frequency. The Mann-Whitney $\mathrm{U}$ test was used for the variables obtained by the measurement and chi-square test for categorical variables. The odds ratio (OR) with $95 \%$ confidence interval (CI) was calculated. Logistic regression analysis was done to identify the effect of group (OA vs. control) in terms of VI for the age adjustment. The effect of factors in dependent samples was evaluated by repeated analysis of two-way analysis of variance (ANOVA). A $p$ value of $<0.05$ was considered statistically significant.

\section{RESULTS}

A total of 118 knees in the knee OA group and 88 knees in the control group were evaluated. Baseline characteristics of the study population are presented in Table 1.

According to the CEAP classification system, the frequency of VI was significantly higher in the OA group $(40.6 \%, \mathrm{n}=24)$ than the control group $(15.9 \%, \mathrm{n}=7)(\mathrm{p}=0.007)$ and 33 (18 right and 15 left) of the 118 lower extremities had VI in the OA group. The risk of VI in the OA group was 3.62-times higher (95\% CI: 1.38-9.43) than normal individuals. However, as the age was significantly higher in the OA group, logistic regression analyses was done. When the effect of age was adjusted, there was not significant difference between $\mathrm{OA}$ and control group in terms of VI $(\mathrm{p}=0.13$, OR: $2.33,95 \% \mathrm{CI}$ : 0.772-7.034).

All patients were between $\mathrm{C} 0-\mathrm{C} 2$ according to clinical category of CEAP. The etiological problem was

\begin{tabular}{|c|c|c|c|c|c|}
\hline \multicolumn{6}{|c|}{$\begin{array}{l}\text { TABLE } 1 \\
\text { Baseline characteristics of study population }\end{array}$} \\
\hline & \multicolumn{2}{|c|}{ OA group $(\mathrm{n}=59)$} & \multicolumn{2}{|c|}{ Control group $(\mathrm{n}=44)$} & \multirow[b]{2}{*}{$p$} \\
\hline & $\%$ & Mean \pm SD & $\%$ & Mean \pm SD & \\
\hline Age (year) & & $52.5 \pm 7.4$ & & $43.2 \pm 6.8$ & $<0.001$ \\
\hline \multicolumn{6}{|l|}{ Gender } \\
\hline Female & 86 & & 86 & & \\
\hline Male & 14 & & 14 & & \\
\hline Body mass index $\left(\mathrm{kg} / \mathrm{m}^{2}\right)$ & & $30 \pm 4.7$ & & $26.7 \pm 4.7$ & \\
\hline Venous insufficiency & 40.6 & & 15.9 & & 0.007 \\
\hline Pain severity & & $2.5 \pm 1.0$ & & - & \\
\hline \multicolumn{6}{|l|}{ WOMAC } \\
\hline Pain $(0-20)$ & & $7.8 \pm 4.4$ & & - & \\
\hline Joint stiffness $(0-8)$ & & $2.2 \pm 1.8$ & & - & \\
\hline Physical function (0-68) & & $26.7 \pm 14.4$ & & - & \\
\hline Total $(0-100)$ & & $36.9 \pm 9.5$ & & - & \\
\hline
\end{tabular}

\begin{tabular}{|c|c|c|c|c|c|c|c|}
\hline \multicolumn{8}{|c|}{$\begin{array}{l}\text { TABLE } 2 \\
\text { Cartilage thickness and Kellgren-Lawrence grading of study population }\end{array}$} \\
\hline & \multicolumn{3}{|c|}{ OA group $(\mathrm{N}=118)$} & \multicolumn{3}{|c|}{ Control group $(\mathrm{N}=88)$} & \multirow[b]{2}{*}{$p$} \\
\hline & Mean \pm SD & Median & Min-Max & Mean \pm SD & Median & Min-Max & \\
\hline Kellgren-Lawrence & & 2 & $0-4$ & & 2 & $0-2$ & $<0.001$ \\
\hline \multicolumn{8}{|l|}{ Cartilage thickness (mm) } \\
\hline Medial femoral condyle & $2.0 \pm 0.7$ & & & $1.9 \pm 0.2$ & & & 0.71 \\
\hline Intercondylar area & $1.9 \pm 0.5$ & & & $2.0 \pm 0.4$ & & & 0.62 \\
\hline Lateral femoral condyle & $1.9 \pm 0.4$ & & & $2.0 \pm 0.8$ & & & 0.89 \\
\hline
\end{tabular}




\begin{tabular}{|c|c|c|c|}
\hline \multicolumn{4}{|c|}{$\begin{array}{l}\text { TABLE } 3 \\
\text { The relation of venous insufficiency with cartilage thickness in extremities of osteoarthritis group } \\
\qquad(\mathrm{N}=118)\end{array}$} \\
\hline & Venous insufficiency $+(\mathrm{N}=33)$ & Venous insufficiency - $(\mathrm{N}=85)$ & \\
\hline Parameter $(\mathrm{mm})$ & Mean \pm SD & Median & $p$ \\
\hline Medial femoral condyle & $2.1 \pm 1.2$ & $1.9 \pm 0.3$ & 0.947 \\
\hline Intercondylar area & $1.9 \pm 0.6$ & $1.9 \pm 0.4$ & 0.770 \\
\hline Lateral femoral condyle & $1.9 \pm 0.3$ & $1.9 \pm 0.4$ & 0.671 \\
\hline
\end{tabular}

\begin{tabular}{|c|c|c|c|c|c|c|c|c|c|}
\hline \multicolumn{10}{|c|}{$\begin{array}{l}\text { TABLE } 4 \\
\text { ency with pain and sclerosis }\end{array}$} \\
\hline & \multicolumn{8}{|c|}{ Osteoarthritis } & \multirow[b]{3}{*}{$p$} \\
\hline & \multicolumn{4}{|c|}{ Venous insufficiency $+(n=24)$} & \multicolumn{4}{|c|}{ Venous insufficiency $-(\mathrm{n}=35)$} & \\
\hline & $\mathrm{N}$ & $\%$ & Median & Min-Max & $\mathrm{N}$ & $\%$ & Median & Min-Max & \\
\hline \multicolumn{10}{|l|}{ WOMAC scores } \\
\hline Pain $(0-20)$ & & & 7.5 & $0-20$ & & & 8.5 & $0-16$ & 0.44 \\
\hline Stiffness $(0-8)$ & & & 1.5 & $0-5$ & & & 3 & $0-7$ & 0.17 \\
\hline Physical functions (0-68) & & & 28.5 & $1-43$ & & & 29 & $0-57$ & 0.32 \\
\hline Total $(0-100)$ & & & 41 & $2-59$ & & & 39 & $0-77$ & 0.30 \\
\hline Likert pain severity (1-5) & & & 3 & $1-5$ & & & 3 & $1-5$ & 0.38 \\
\hline Presence of sclerosis (extremities) & 20 & 60.6 & & & 44 & 51.8 & & & 0.38 \\
\hline Kellgren-Lawrence grading & & & 2 & $0-4$ & & & 2 & $0-4$ & 0.96 \\
\hline
\end{tabular}

primary VI and the main pathophysiological problem was reflux in all of the patients. None of the patients had a history of deep venous thrombosis.

The K\&L staging was Stage $\leq 2$ in $78 \%$ of patients in the OA group. The cartilage thickness of the knees of OA and control group was similar (Table 2). The cartilage thickness of the patients with VI in the OA group were found to be similar to those without VI (Table 3).

The K\&L grading, WOMAC pain, and Likert pain severity scores were similar in patients, regardless of the presence of VI in OA group ( $p>0.05$ ). Twenty $(60.6 \%)$ of the extremities of OA patients with VI had radiographic medial tibial sclerosis ( $\mathrm{p}>0.05)$ (Table 4).

The presence of deep VI was detected in 21 lower extremities among all participants in the study and 16 of these extremities (78\%) were in the knee OA group. Patients with deep VI had higher WOMAC pain scores (9.2 \pm 6.4 ; median: 10 ; range, 0 to 20 ) than those without deep VI ( $7.5 \pm 4.2$; median: 8 ; range, 0 to 16$)$ in the knee OA group $(\mathrm{p}>005)$.

\section{DISCUSSION}

In the present study, we examined a possible relationship between VI and knee OA. Our study results showed that $40.6 \%$ of the OA group and $15.9 \%$ of the control group had VI.

It has been shown that patients with knee OA are reported to develop symptoms of chronic VI more common than their healthy peers. ${ }^{[6]}$ However, VI and OA share common risk factors and the risk of these diseases increases with age. ${ }^{[13]}$ Our results showed that the difference in the frequency of VI between the groups was not remained after the age adjustment.

In our study, $78 \%$ of the patients in the knee OA group were $\leq$ Grade 2 according to the K\&L staging. The K\&L grading evaluates knees according to JSN and presence of osteophyte. ${ }^{[10]}$ Ultrasonography has been used for measuring cartilage thickness in knee $\mathrm{OA}$ recently and has been shown to be valuable as magnetic resonance imaging (MRI) for this purpose. ${ }^{[14]}$ In our study, knee OA was detected on weight-bearing knee radiographs; however, no significant decrease in the cartilage thickness was found between the 
control and OA groups. Agnesi et al. ${ }^{[15]}$ evaluated knee articular cartilage thickness by MRI according to knee OA stage and they were unable to find any statistically significant decrease in the cartilage thickness between Grade 1 and Grade 2 OA. The authors reported that significant decrease started after Grade 2. This may be one of the reasons why we were unable to find any significant difference in the cartilage thickness between the control and OA groups. However, it is known that MRI changes in the subchondral bone in early $\mathrm{OA}^{[16]}$ suggest that $\mathrm{OA}$ is not always a process starting from articular cartilage, but a bone disease that affects bone structure and bone remodeling. In our study, the patients with OA had no distinct JSN, but had osteophytic changes on plain radiographs. Although knee OA was detected radiographically, the absence of thinning in the cartilage thickness may be due to the fact that the subchondral bone involvement starts earlier in the etiopathogenesis of OA.

In our study, cartilage thicknesses were similar in the OA group, irrespective of VI. However, $60 \%$ of knee OA patients with, VI presented radiographic medial tibial sclerosis on direct radiographs. $18-\mathrm{F}$ positron emission tomography and dynamic susceptibility contrast-MRI were used to quantify perfusion characteristics and determine changes in subchondral bone perfusion changes in animal models. ${ }^{[17]}$ A study was conducted in Dunkin-Hartley guinea pigs to show perfusion abnormalities in knee OA models. ${ }^{[17]}$ It was shown that the venous outflow obstruction in medial tibiofemoral compartment had positive correlation with severity of OA. Increased medial tibial sclerosis in our findings could be related to this process. Another possible reason is that intraosseous hypertension due to vascular changes in bone alters the mechanical properties and leads to the reduced ability of the bone to act as a shock absorber. ${ }^{[18]}$ This process can result in microcracks and consequently bone resorption and formation, failure of bone healing, and increased subchondral sclerosis.

Bone veins play an important role in bone resorption and formation. ${ }^{[19]}$ Møller et al. ${ }^{[3]}$ examined the relationship of the vessels with the trabecular bone and showed that the blood vessels were mostly seen in the bone resorption sites. In addition, Wang et al. ${ }^{[4]}$ evaluated the adaptation of bone to venous stasis and suggested that venous stasis increased intramedullary pressure, leading to increased periosteal bone production by irritating the periosteum. They suggested that the metabolic changes due to venous congestion, such as oxygen, carbon dioxide tension and local $\mathrm{pH}$ value might cause the periosteal response. Also, they emphasized that an increase on extravascular fluid pressure in response to venous stasis might pressurize the periosteum, leading to periosteal deformity and play a role in the periosteal new bone growth. These findings suggest us the possibility of affected medial tibial microenvironment by VI in the knee OA group. Degeneration process could start from the subchondral bone, leading to the formation of osteophytes by increasing the periosteal bone formation before decreasing the joint cartilage thickness.

In our study, there was no significant difference in the WOMAC pain scores between the OA patients with and without VI. Ay et al. ${ }^{[20]}$ examined the association of VI in patients with lower extremity pain and its effects on pain and functional capacity. Venous insufficiency was found in 33\% of patients with knee OA and the authors found no statistically significant difference in the WOMAC scores of patients, similar to our findings. On the other hand, Kiaer $^{[21]}$ reported that elevated intraosseous venous pressure resulted in an increased substance $\mathrm{P}$, leading to increased bone pain. Also, Arnoldi et al. ${ }^{[22]}$ showed that the increased pressure in the internal saphenous vein was associated with resting pain in OA. Simkin ${ }^{[23]}$ reported that resting pain in knee and hip in patients with gonarthrosis and coxarthrosis was due to intraosseous hypertension and also emphasized that the mechanism of this process was not fully understood, although intraosseous phlebography implicated outflow impairment in the relatively distal venous sites. The authors, hence, concluded that increased outflow resistance might amplify the episodic pressure response with subsequent intravasation of epiphyseal fat leading to marrow edema and altered mechanics. ${ }^{[23]}$ When the WOMAC pain scores were evaluated according to deep vein involvement in OA group, the patients with deep VI had higher WOMAC pain scores in our study. Considering the fact that deep venous system involvement increases the risk of intraosseous hypertension more than superficial venous system involvement, our findings are consistent with the literature. Future studies are important to show whether there is a relationship between intraosseous hypertension and VI in patients with OA.

There are several limitations to this study. First, the mean age of the OA group was higher than the control group, as OA is more common in elderly people. Second, knee OA patients were unable to be 
evaluated by MRI which is a good option to show whether early subchondral bone changes is present in patients with VI. Also, the fact that venous pressure measurement was not performed by venous phlebography is another limitation. However, this process is an invasive method and may not be appropriate for the study participants due to ethical considerations. Third, power analysis was unable to be performed before the study, due to the lack of a similar study in the literature. Therefore, further studies including patients with deep VI and using MRI may provide more accurate results.

In conclusion, to the best of our knowledge, this is the first study to evaluate possible relationships between VI and radiographic changes in knee OA. Our study results suggest that venous system pathologies can affect the intraosseous microenvironment of the bone and, therefore, subchondral bone involvement and consequently subchondral sclerosis may manifest earlier than articular cartilage involvement. We believe that future studies, overcoming the limitations of the present study, would provide a better understanding of the role of VI in the pathogenesis of OA.

\section{Declaration of conflicting interests}

The authors declared no conflicts of interest with respect to the authorship and/or publication of this article.

\section{Funding}

The authors received no financial support for the research and/or authorship of this article.

\section{REFERENCES}

1. Manara M, Varenna M. A clinical overview of bone marrow edema. Reumatismo 2014;66:184-96.

2. Uysal FG, Başaran S. Knee Osteoarthritis. Turk J Phys Med Rehab 2009:55;1-7.

3. Møller JF, Robertsen K, Bünger C, Hansen ES. Improved method for examination of microvascular structures in bone tissue. Clin Orthop Relat Res 1997;334:15-23.

4. Wang L, Fritton SP, Weinbaum S, Cowin SC. On bone adaptation due to venous stasis. J Biomech 2003;36:1439-51.

5. Hayami T, Pickarski M, Zhuo Y, Wesolowski GA, Rodan GA, Duong LT. Characterization of articular cartilage and subchondral bone changes in the rat anterior cruciate ligament transection and meniscectomized models of osteoarthritis. Bone 2006;38:234-43.

6. Lesnyak OM, Zubareva EV, Goncharova MG, Maksimov DM. Lower extremity venous diseases in primary knee osteoarthritis. Ter Arkh 2017;89:53-9.

7. Altman R, Asch E, Bloch D, Bole G, Borenstein D, Brandt $\mathrm{K}$, et al. Development of criteria for the classification and reporting of osteoarthritis. Classification of osteoarthritis of the knee. Diagnostic and Therapeutic Criteria Committee of the American Rheumatism Association. Arthritis Rheum 1986;29:1039-49.

8. Labropoulos N, Tiongson J, Pryor L, Tassiopoulos AK, Kang SS, Ashraf Mansour M, et al. Definition of venous reflux in lower-extremity veins. J Vasc Surg 2003;38:793-8.

9. Porter JM, Moneta GL. Reporting standards in venous disease: an update. International Consensus Committee on Chronic Venous Disease. J Vasc Surg 1995;21:635-45.

10. Kellgren JH, Lawrence JS. Radiological assessment of osteoarthrosis. Ann Rheum Dis 1957;16:494-502.

11. Özçakar L, Kara M, Chang KV, Tok F, Hung CY, Akkaya N, et al. EURO-MUSCULUS/USPRM. Basic scanning protocols for knee. Eur J Phys Rehabil Med 2015;51:641-6.

12. Tüzün EH, Eker L, Aytar A, Daşkapan A, Bayramoğlu M. Acceptability, reliability, validity and responsiveness of the Turkish version of WOMAC osteoarthritis index. Osteoarthritis Cartilage 2005;13:28-33.

13. Evans CJ, Fowkes FG, Ruckley CV, Lee AJ. Prevalence of varicose veins and chronic venous insufficiency in men and women in the general population: Edinburgh Vein Study. J Epidemiol Community Health 1999;53:149-53.

14. Schmitz RJ, Wang HM, Polprasert DR, Kraft RA, Pietrosimone BG. Evaluation of knee cartilage thickness: A comparison between ultrasound and magnetic resonance imaging methods. Knee 2017;24:217-23.

15. Agnesi F, Amrami KK, Frigo CA, Kaufman KR. Comparison of cartilage thickness with radiologic grade of knee osteoarthritis. Skeletal Radiol 2008;37:639-43.

16. Felson DT, Chaisson CE, Hill CL, Totterman SM, Gale $\mathrm{ME}$, Skinner KM, et al. The association of bone marrow lesions with pain in knee osteoarthritis. Ann Intern Med 2001;134:541-9.

17. Dyke JP, Synan M, Ezell P, Ballon D, Racine J, Aaron RK. Characterization of bone perfusion by dynamic contrastenhanced magnetic resonance imaging and positron emission tomography in the Dunkin-Hartley guinea pig model of advanced osteoarthritis. J Orthop Res 2015;33:366-72.

18. Conaghan PG, Vanharanta H, Dieppe PA. Is progressive osteoarthritis an atheromatous vascular disease? Ann Rheum Dis 2005;64:1539-41.

19. Parfitt AM. The mechanism of coupling: a role for the vasculature. Bone 2000;26:319-23.

20. Ay S, Evcik D, Koldaş Doğan Ş. Alt ekstremite ağrısı olan hastalarda venöz yetmezlik birlikteliği, ağrı ve fonksiyonel kapasite üzerine etkisi. Yeni Tip Dergisi 2010;27:18-21.

21. Kiaer T, Grønlund J, Sørensen KH. Intraosseous pressure and partial pressures of oxygen and carbon dioxide in osteoarthritis. Semin Arthritis Rheum 1989;18:57-60.

22. Arnoldi CC, Lemperg K, Linderholm H. Intraosseous hypertension and pain in the knee. J Bone Joint Surg [Br] 1975;57:360-3.

23. Simkin PA. Bone pain and pressure in osteoarthritic joints. Novartis Found Symp 2004;260:179-86. 